\title{
Friction of a Brownian Particle in a Lennard-Jones Solvent: A Molecular Dynamics Simulation Study
}

\author{
Song Hi Lee \\ Department of Chemistry, Kyungsung University, Busan 608-736, Korea \\ Received January 9, 2010, Accepted March 3, 2010
}

\begin{abstract}
In this work, equilibrium molecular dynamics (MD) simulations in a microcanonical ensemble are performed to evaluate the friction coefficient of a Brownian particle (BP) in a Lennard-Jones (LJ) solvent. The friction coefficients are determined from the time dependent friction coefficients and the momentum autocorrelation functions of the $\mathrm{BP}$ with its infinite mass at various ratios of LJ size parameters of the $\mathrm{BP}$ and solvent, $\sigma_{\mathrm{B}} / \sigma_{\mathrm{s}}$. The determination of the friction coefficients from the decay rates of the momentum autocorrelation functions and from the slopes of the time dependent friction coefficients is difficult due to the fast decay rates of the correlation functions in the momentumconserved MD simulation and due to the scaling of the slope as $1 / \mathrm{N}(\mathrm{N}$ : the number of the solvent particle), respectively. On the other hand, the friction coefficient can be determined correctly from the time dependent friction coefficient by measuring the extrapolation of its long time decay to $t=0$ and also from the decay rate of the momentum autocorrelation function, which is obtained by time integration of the time dependent friction coefficient. It is found that while the friction coefficient increases quadratically with the ratio of $\sigma_{\mathrm{B}} / \sigma_{\mathrm{s}}$ for all $\sigma_{\mathrm{B}}$, for a given $\sigma_{\mathrm{s}}$ the friction coefficient increases linearly with $\sigma_{\mathrm{B}}$.
\end{abstract}

Key Words: Friction, Brownian particle, Lennard-Jones solvent, Molecular dynamics simulation

\section{Introduction}

Understanding friction and measuring the friction coefficients of molecules in liquids are basic problems that can be used to characterize the dynamics of solute molecules in solution. The friction coefficient plays a central role in problems ranging from the rates of chemical reactions in the condensed phase to the description of polymer dynamics in solution. The force and momentum autocorrelation functions from which the friction coefficient may be determined present interesting features. The computation of the friction coefficient from the time integral of the force autocorrelation function must deal with the plateau value problem since the infinite time integral of this correlation function is zero. ${ }^{1}$ The only way to have a non-zero value for the friction coefficient is by first taking the infinite mass limit of the solute. On the other hand, the calculation of the friction coefficient from the momentum autocorrelation must account for the exponential decay of this quantity at long times due to the evolving of the solvent particles in the presence of the external force field generated by the fixed particle.

Calculations of the friction coefficient by molecular dynamics (MD) simulations are well-studied problems that have been addressed many times. Nevertheless, the computation of the friction coefficient from MD simulations involves a number of subtle issues for finite-size systems as discussed in several recent papers. $^{2-4}$ The problems center around the definition of the friction coefficient in terms of the projected dynamics and its relation to the fixed-particle friction coefficient for a massive Brownian particle (BP). The estimates of the friction coefficients have been shown to depend on the order in which the mass of the $\mathrm{BP}$ and the solvent particle number $\mathrm{N}$ are taken to infinity. For finite-size systems one must investigate how large $\mathrm{N}$ must be to obtain a reliable estimate of the friction; this typi- cally requires very large MD simulations. Similarly, the estimate of the diffusion coefficient from the velocity autocorrelation function requires large scale simulations, especially for large BPs, due to the importance of hydrodynamic contributions.

Recently the friction and diffusion coefficients of a massive $\mathrm{BP}$ in a mesoscopic solvent were computed from the force and velocity autocorrelation functions. ${ }^{5}$ The mesoscopic solvent is described in terms of free streaming of the solvent molecules, interrupted at discrete time intervals by multi-particle collisions that conserve mass, momentum, and energy. The BP interacts with the solvent molecules through repulsive Lennard-Jones forces. The decays of the force and velocity correlation functions are analyzed in the microcanonical ensemble as a function of the number $\mathrm{N}$ of solvent molecules and the BP mass and diameter. The simulations were carried out for large system sizes and long times to assess the N-dependence of the friction coefficient. The decay rates of these correlations are confirmed to vary as $\mathrm{N}^{-1}$ in accord with earlier predictions. ${ }^{4}$

The purpose of this paper is twofold: First, we investigate the issue discussed above by carrying out large scale simulations confirming the treatment of momentum conservation of the whole system related to the estimates of the friction coefficients. Second, we use these calculations to understand the effects of the size ratio of the $\mathrm{BP}$ and the solvent particle on the friction coefficient.

The outline of the paper is as follows. In Sec. II we specify the system being investigated and also sketch the molecular dynamics simulation methods. The friction coefficients that are of interest in this study are defined in Sec. III. In Sec. IV we present the results of simulations of the friction coefficients for a smooth spherical BP interacting with the solvent particles through purely repulsive Lennard-Jones forces. The conclusions of the paper are given in Sec. V. 


\section{Molecular Dynamics Simulation Details}

There are 9 Lennard-Jones (LJ) systems under investigation, as shown in Table 1. Each system consists of a BP with LJ parameter $\sigma_{\mathrm{B}}$ and solvent particles of $\mathrm{N}=32,000$ each of which has LJ parameter $\sigma_{\mathrm{s}}$. The LJ potential used in our molecular dynamics (MD) simulations is a purely repulsive CWA (Chandler-Weeks-Andersen) potential: ${ }^{6}$

$$
\mathrm{v}^{\mathrm{CWA}}(\mathrm{r})= \begin{cases}4 \in\left[\left(\frac{\sigma}{\mathrm{r}}\right)^{12}-\left(\frac{\sigma}{\mathrm{r}}\right)^{6}+\frac{1}{4}\right], & \mathrm{r} \leq \mathrm{r}_{\mathrm{c}} \\ 0, & \mathrm{r}>\mathrm{r}_{\mathrm{c}}\end{cases}
$$

where the LJ parameters are chosen as $\sigma=\left(\sigma_{\mathrm{B}}+\sigma_{\mathrm{s}}\right) / 2$ for the $\mathrm{BP}$-solvent particle interaction or $\sigma=\sigma_{\mathrm{s}}$ for interaction between solvent particles and $\varepsilon=1.006 \mathrm{~kJ} / \mathrm{mol}$. The inter-particle potential is truncated at $r_{c}=2^{1 / 6} \sigma$. The masses of the LJ particles are $\mathrm{m}=3.995 \mathrm{~g} / \mathrm{mol}$ which is $1 / 10$ of argon mass. The mass of the BP is infinity and several MD simulation methods are employed for the treatment of the infinite mass limit in Sec. IV. The length of the simulation box is obtained from the fixed reduced density of the system which is defined as $\rho^{*}=\rho \sigma_{\mathrm{s}}{ }^{3}=$ $\mathrm{N \sigma}_{\mathrm{s}}{ }^{3} / \mathrm{V}$. The value of $\rho^{*}$ is chosen as 0.8 and the lengths of the simulation boxes are given in Table 1 . The preliminary canonical ensemble (NVT fixed) MD simulations of $\mathrm{N}=32,001 \mathrm{LJ}$ particles are started in the cubic box of given lengths with a fixed temperature $\mathrm{T}=40.33 \mathrm{~K}$ and then the simulations are switched to the microcanonical ensemble (NVE fixed) after a long time equilibration for the constant temperature. The equations of motion are solved using the velocity Verlet algorithm ${ }^{7}$ with a time step of $2 \times 10^{-15}$ seconds. All the systems were fully equilibrated and the equilibrium properties are averaged over 50 blocks of 1,000,000 time steps. The configurations of the BP were stored every 5 time steps for further analyses.

\section{Force and Momentum Autocorrelation Functions}

The friction coefficient is given in terms of the integration on time $t$ of the equilibrium autocorrelation function $\langle\mathbf{F}(\mathrm{t}) \cdot \mathbf{F}(0)\rangle$ of the instantaneous microscopic force $\mathbf{F}(\mathrm{t})$ experienced by the BP in the well-known Green-Kubo formula: ${ }^{8}$

Table 1. Molecular dynamics simulation parameters for several Lennard-Jones (LJ) systems

\begin{tabular}{cccc}
\hline System & $\begin{array}{c}\sigma_{\mathrm{B}} \text { of Brownian } \\
\text { particle }(\mathrm{nm})\end{array}$ & $\begin{array}{c}\sigma_{\mathrm{S}} \text { of LJ solvent } \\
\text { particle }(\mathrm{nm})\end{array}$ & $\begin{array}{c}\text { Length of } \\
\text { box }(\mathrm{nm})\end{array}$ \\
\hline $\mathrm{S} 2 / 1$ & 0.2 & 0.1 & 3.42 \\
$\mathrm{~S} 2 / 2$ & 0.2 & 0.2 & 6.84 \\
$\mathrm{~S} 2 / 4$ & 0.2 & 0.4 & 13.68 \\
$\mathrm{~S} 4 / 2$ & 0.4 & 0.2 & 6.84 \\
$\mathrm{~S} 4 / 4$ & 0.4 & 0.4 & 13.68 \\
$\mathrm{~S} 4 / 8$ & 0.4 & 0.8 & 27.36 \\
$\mathrm{~S} 8 / 4$ & 0.8 & 0.4 & 13.68 \\
$\mathrm{~S} 8 / 8$ & 0.8 & 0.8 & 27.36 \\
$\mathrm{~S} 8 / 16$ & 0.8 & 1.6 & 54.72
\end{tabular}

$$
\zeta=\frac{1}{3 \mathrm{kT}} \int_{0}^{\infty} \mathrm{dt}<\mathbf{F}(\mathrm{t}) \cdot \mathbf{F}(0)>,
$$

where $\mathrm{k}$ is Boltzmann's constant and $\mathrm{T}$ is the absolute temperature. However, for finite size systems, it is expected that, for $\mathrm{t} \rightarrow \infty$, the friction coefficient should be zero according to the ergodic postulate of equilibrium statistical mechanics. ${ }^{1}$

On the other hand, using the projection operator technique ${ }^{9-12}$ it is possible to derive an alternate expression for the timedependent friction coefficient:

$$
\zeta=\frac{1}{3 \mathrm{kT}} \int_{0}^{\infty} \mathrm{dt} \lim _{\mathrm{M} \rightarrow \infty}<\mathbf{F}(\mathrm{t}) \cdot \mathbf{F}(0)>,
$$

where $\mathrm{M}$ is the mass of the BP. It is convenient to define a time dependent friction coefficient as the finite time integral of the projected force, $\mathbf{F}^{+}(\mathrm{t})$, autocorrelation function as,

$$
\zeta(\mathrm{t})=\frac{1}{3 \mathrm{kT}} \int_{0}^{\mathrm{t}} \mathrm{d} \tau<\mathbf{F}^{+}(\tau) \cdot \mathbf{F}(0)>.
$$

Through the Laplace transforms of the projected and unprojected force autocorrelation functions, ${ }^{1}$ the time dependent friction coefficient is simply given by

$$
\zeta_{\mathrm{u}}(\mathrm{t}) \approx \zeta^{-\zeta \mathrm{t} / \mu}
$$

where $\mu=\mathrm{MNm} /(\mathrm{M}+\mathrm{Nm})$ and $\mu \rightarrow \mathrm{Nm}$ in the limit $\mathrm{M} \rightarrow \infty$ with the number of solvent particles, $\mathrm{N}$, and the mass of the solvent particle, m. According to Eq. (5), we can estimate the friction coefficient in two ways: the extrapolation of the long time decay of the time dependent friction coefficient $\zeta_{\mathrm{u}}(\mathrm{t})$ to $\mathrm{t}=$ 0 (noted as $\zeta_{1}$ ), or from the decay rates of the force autocorrelation function or $\zeta_{\mathrm{u}}(\mathrm{t})$ (noted as $\zeta_{2}$ ). In our MD simulations the actual expression to calculate the time dependent friction coefficient is given in terms of the unprojected force autocorrelation function

$$
\zeta_{\mathrm{u}}(\mathrm{t})=\frac{1}{3 \mathrm{kT}} \int_{0}^{\mathrm{t}} \mathrm{d} \tau \lim _{\mathrm{M} \rightarrow \infty}<\mathbf{F}(\tau) \cdot \mathbf{F}(0)>
$$

The momentum autocorrelation function can be determined from the Langevin equation and decays exponentially as

$$
\mathrm{C}(\mathrm{t})=<\mathbf{P}(\mathrm{t}) \cdot \mathbf{P}(0)>/<\mathbf{P}(0)^{2}>=\mathrm{e}^{-\zeta \mathrm{t} / \mu}
$$

Since the diffusion coefficient is the infinite time integral of the velocity correlation function we have the Einstein relation, $\mathrm{D}=\mathrm{kT} / \zeta$. The friction coefficient can be determined from the long time decay of the momentum autocorrelation function (noted as $\zeta_{3}$ ) according to Eq. (7).

It is interesting that for long times the other form of $\zeta_{\mathrm{u}}(\mathrm{t})$ in Eq. (6) can be obtained by the time derivative of $C(t)$ in Eq. (7): 


$$
\begin{aligned}
& -\mathrm{Nm} \frac{\mathrm{d}}{\mathrm{dt}} \mathrm{C}(\mathrm{t})=\frac{1}{3 \mathrm{kT}}<\mathbf{F}(\mathrm{t}) \cdot \mathbf{P}(0)> \\
& =\frac{1}{3 \mathrm{kT}} \lim _{\mathrm{s} \rightarrow \infty} \frac{1}{\mathrm{~s}} \int_{0}^{\mathrm{s}} \mathrm{du} \mathbf{F}(\mathrm{u}+\mathrm{t}) \cdot \mathbf{P}(\mathrm{u}) \\
& =-\frac{1}{3 \mathrm{kT}} \lim _{\mathrm{s} \rightarrow \infty} \frac{1}{\mathrm{~s}} \int_{0}^{\mathrm{s}} \mathrm{du} \mathbf{F}(\mathrm{u}) \cdot \mathbf{P}(\mathrm{u}+\mathrm{t}) \\
& =\frac{1}{3 \mathrm{kT}} \lim _{\mathrm{s} \rightarrow \infty} \frac{1}{\mathrm{~s}} \int_{0}^{\mathrm{s}} \mathrm{du} \int_{0}^{\mathrm{t}} \mathrm{d} \tau \mathbf{F}(\mathrm{u}) \cdot \mathbf{F}(\mathrm{u}+\tau) \\
& =\frac{1}{3 \mathrm{kT}} \int_{0}^{\mathrm{t}} \mathrm{d} \tau<\mathbf{F}(\tau) \cdot \mathbf{F}(0)>=\xi_{\mathrm{u}}(\mathrm{t}),
\end{aligned}
$$

where $<\mathbf{P}(0)^{2}>=3 \mathrm{kTNm}$ in the limit $\mathrm{M} \rightarrow \infty, \mathrm{P}(\mathrm{t})=-\int_{0}^{\mathrm{t}} \mathrm{d} \tau \mathbf{F}(\tau)$ was used and $\xi_{\mathrm{u}}(\mathrm{t})$ was also used to distinguish from $\zeta_{\mathrm{u}}(\mathrm{t})$ in Eq. (6), and vice versa:

$$
1-\frac{1}{\mathrm{Nm}} \int_{0}^{\mathrm{t}} \mathrm{d} \tau \zeta_{\mathrm{u}}(\tau)=\mathrm{c}(\mathrm{t})
$$

where $\mathrm{c}(\mathrm{t})$ was used to distinguish $\mathrm{C}(\mathrm{t})$ in Eq. (7). By time differentiation of $\mathrm{C}(\mathrm{t})$ in Eq. (8), two more friction coefficients are obtained from $\xi_{\mathrm{u}}(\mathrm{t})$ (noted as $\zeta_{4}$ and $\zeta_{5}$ ), like $\zeta_{1}$ and $\zeta_{2}$ in Eq. (5), and furthermore by time integration of $\zeta_{\mathrm{u}}(\mathrm{t})$ in Eq. (9), one more friction coefficient is obtained from $\mathrm{c}(\mathrm{t})$ (noted as $\left.\zeta_{6}\right)$, like $\zeta_{3}$ in Eq. (7). Hence, we are dealing with a total of 6 friction coefficients.

\section{Results and Discussion}

In the definition of the friction coefficient three limit procedures are involved; the long time limit $(\mathrm{t} \rightarrow \infty)$; the thermodynamic limit $(\mathrm{N} \rightarrow \infty)$; and the infinite mass limit $(\mathrm{M} \rightarrow \infty){ }^{3}$ The ordering of these limits is very important as they do not always commute. This can be easily observed in the expression (5) for the time dependent friction coefficient in the Langevin approximation. The Langevin approximation is expected to be valid for a finite but sufficiently large mass of the BP and for a large number of solvent particles. If we first consider the infinite time limit the resulting friction coefficient is zero. The only way to have a non-zero value for the friction coefficient is by first taking $\mathrm{M} \rightarrow \infty$. ${ }^{3}$ In the thermodynamic limit $\mathrm{N} \rightarrow \infty$, the projected and unprojected force autocorrelation functions are the same ${ }^{1,5}$ and the expression (6) is possible. Since MD simulations are carried out at finite $\mathrm{N}$, the study of the $\mathrm{N}$ (and $\mathrm{M})$ dependence of $\zeta_{\mathrm{u}}(\mathrm{t})$ and the estimate of the friction coefficient from either the decay of the momentum or force autocorrelation functions is of interest. ${ }^{4,5}$

Furthermore, in order to calculate the friction coefficients of the BP from Eqs. (6) and (7), the mass of the BP, M, becomes infinity, or the BP is fixed in space using a holonomic constraint method. ${ }^{13}$ While the MD simulation by using an infinite mass violates the equation of motion since the BP never moves with the force on it, the constraint method MD simulation returns the BP back to its original position with zero velocity, and trajectories by both MD simulations are not the same. However, it is found that the momenta of the whole system carried out by both the infinite mass and the constraint method MD simulations are not conserved, because the momentum of the BP is not well defined with zero velocity and infinite mass. Nevertheless, the momentum of the fixed particle is defined as the negative of the total momentum of the solvent particles. ${ }^{3,4}$ In Fig. 1, the logarithm of the normalized momentum auto correlation functions, $\mathrm{C}(\mathrm{t})$ and $\mathrm{c}(\mathrm{t})$, is shown for different $\mathrm{MD}$ simulations and using Eq. (9) for the system of S2/2. The momentum autocorrelation functions obtained from both the infinite mass and the constraint method MD simulations in this plot are not linear, and the obtained values of $\zeta_{3}$ according to Eq. (7) under the assumption of the linearity of these functions are 1.080 $\mathrm{kg} /(\mathrm{mol} \cdot \mathrm{ps}$ ) by the infinite mass MD and $0.924 \mathrm{~kg} /(\mathrm{mol} \cdot \mathrm{ps})$ by the constraint method MD, respectively.

A reasonable trick to bypass this difficulty is to put the mass of the BP as $10^{90} \mathrm{~g} / \mathrm{mol}$, and in this case the momentum of the system is conserved: The magnitude of the mass of the BP is on the order of 90 and its velocity is on the order of -90 , but its momentum has a finite value and is equal to the negative of the total momentum of the solvent particles. However, the total instantaneous momentum of the whole system is on the order of $-5 \sim-6$. The linear behavior of the momentum autocorrelation function obtained from the momentum-conserved MD simulation in Fig. 1 indicates an exponential decay and the estimate of $\zeta_{3}$ from the slope of the straight line is $0.588 \mathrm{~kg} /$ (mol-ps). The momentum autocorrelation function obtained from the momentum-conserved MD simulation is very different from those obtained from both the infinite mass and the constraint method MD simulations.

On the other hand, the time dependent friction coefficients for different MD simulations for the system of S2/2 are plotted in Fig. 2. While the time dependent friction coefficient obtained from the momentum-conserved MD simulation fluctuates along the least-square fit straight line (the better view is given in Fig. 3 as the dotted line), the other two coefficients obtained

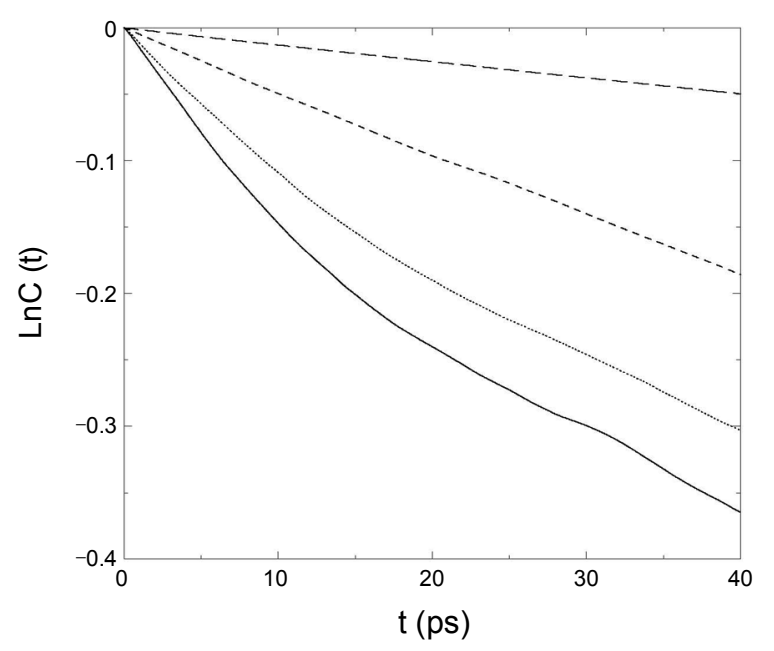

Figure 1. Logarithm of the normalized momentum autocorrelation functions for the system of $\mathrm{S} 2 / 2$. Solid line, the infinite mass MD simulation; dotted line, the constraint MD simulation; dashed line, the momentum-conserved MD simulation; and long-dashed line, $\mathrm{c}(\mathrm{t})$ obtained by time integration of $\zeta_{\mathrm{u}}(\mathrm{t})$, which is obtained from the momentum-conserved MD simulation, using Eq. (9). 


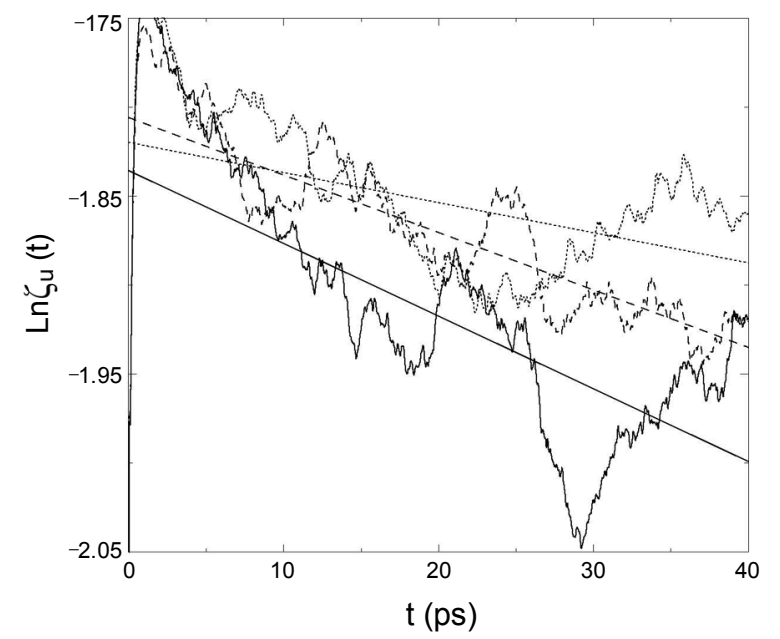

Figure 2. Logarithm of the time dependent friction coefficients for the system of S2/2. Solid line, the infinite mass MD simulation; dotted line, the constraint MD simulation; and dashed line, the momentumconserved MD simulation. The straight lines show the extrapolation of the exponential long time-decay to $t=0$ to determine the value of $\zeta_{1}$.

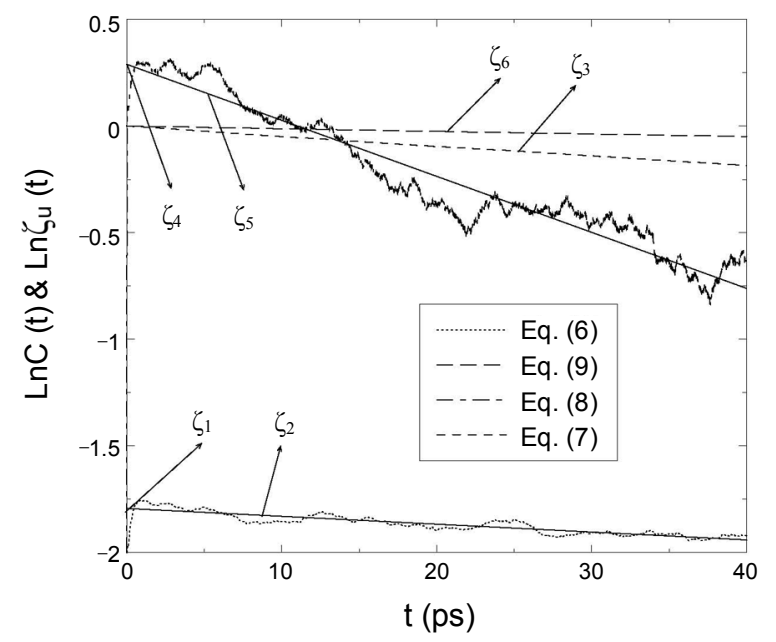

Figure 3. Logarithm of the normalized momentum autocorrelation functions and time dependent friction coefficients for the system of $\mathrm{S} 2 / 2$. Dotted line, $\zeta_{\mathrm{u}}(\mathrm{t})$ calculated directly from Eq. (6); dashed line, $\mathrm{C}(\mathrm{t})$ calculated directly from Eq. (7); dot-dashed line, $\xi_{\mathrm{u}}(\mathrm{t})$ obtained by time differentiation of the dashed line $C(t)$ using Eq. (8); and long-dashed line, $\mathrm{c}(\mathrm{t})$ obtained by time integration of the dotted line $\zeta_{u}(t)$ using Eq. (9). The straight solid lines are the extrapolation of the exponential long time-decay to $\mathrm{t}=0$ to determine $\zeta$ 's.

from both the infinite mass and the constraint MD simulations deviate from the corresponding straight lines. The $\zeta_{1}$ values estimated from the extrapolation of the long time decay of the time dependent friction coefficient $\zeta_{u}(t)$ to $t=0$ are $0.160,0.162$, and $0.167 \mathrm{~kg} /(\mathrm{mol} \cdot \mathrm{ps})$, respectively. In general, these values are the same within statistical error. The total instantaneous force of the whole system for the three MD simulations is equally on the order of $-13 \sim-14$. The momentum autocorrelation function $\mathrm{c}(\mathrm{t})$ obtained by time integration of $\zeta_{\mathrm{u}}(\mathrm{t})$, which is calculated from the momentum-conserved MD simulation, using Eq. (9) is plotted as the long-dashed line in Fig. 1 and the estimate of $\zeta_{6}$ is $0.158 \mathrm{~kg} /(\mathrm{mol} \cdot \mathrm{ps})$. The agreement of this value of $\zeta_{6}$ with the above values of $\zeta_{1}$ is natural since the calculation of the time dependent friction coefficients $\zeta_{\mathrm{u}}(\mathrm{t})$ in Eq. (6) for the three MD simulations is valid, and the momentum autocorrelation function $\mathrm{c}(\mathrm{t})$ is obtained by time integration of $\zeta_{\mathrm{u}}(\mathrm{t})$.

Comparing the slopes, $-\zeta / \mathrm{Nm}$, of the four momentum autocorrelation functions $\mathrm{C}(\mathrm{t})$ and $\mathrm{c}(\mathrm{t})$ in Fig. $1\left(\zeta_{3}=1.080,0.924\right.$, 0.588 , and $\left.\zeta_{6}=0.158 \mathrm{~kg} /(\mathrm{mol} \cdot \mathrm{ps})\right)$, the three $\mathrm{C}(\mathrm{t})$ values calculated directly from the momentum of the BP using Eq. (7) are wrong. The main problem is the momentum conservation of the system. In the case of the momentum-conserved MD simulation, the total instantaneous momentum of the whole system is on the order of $-5 \sim-6$, even when using the double precision code. This might be related to the well-known rounding error in the calculation of the velocity in the usual MD simulation. The next possible explanation for this is related to the thermodynamic limit $(\mathrm{N} \rightarrow \infty)$. In our MD simulation, since $\mathrm{N}=32,000$ and $\mathrm{Nm}=127.84 \mathrm{~kg} / \mathrm{mol}$, the slope, $-\zeta / \mathrm{Nm}$, of the logarithm of $\mathrm{C}(\mathrm{t})$ should be -0.00125 ps to get $\zeta=0.16 \mathrm{~kg} /$ (mol.ps), which means that, at $\mathrm{t}=40 \mathrm{ps}, \ln \mathrm{C}(\mathrm{t})=-0.05$ and $\mathrm{C}(\mathrm{t})=0.95$. Hence, the decay of $\mathrm{C}(\mathrm{t})$ should be very small even after $40 \mathrm{ps}$. Since $-\zeta / \mathrm{Nm}$ scales as $\mathrm{N}^{-1}, \mathrm{C}(\mathrm{t})$ should decay very slowly for large $\mathrm{N}$. However, the actual value of $\ln \mathrm{C}(\mathrm{t})$ at $\mathrm{t}=$ $40 \mathrm{ps}$ is -0.186 as seen in Fig. 1 .

In the previous mesoscopic MD simulation study ${ }^{5}$ the systems were such that $\mathrm{N}=5,120 \sim 327,680, \mathrm{M} / \mathrm{m}=5 \mathrm{~N} \sim 200 \mathrm{~N}$ and $\mathrm{M}=\infty$. The obtained friction coefficients of the BP from $\mathrm{C}(\mathrm{t})$ and $\zeta_{\mathrm{u}}(\mathrm{t})$ were almost the same for various values of $\mathrm{N}$ and $\mathrm{M}$, which means the calculated $\mathrm{C}(\mathrm{t})$ were correct, and the decay of $\mathrm{C}(\mathrm{t})$ scaled very well as $\mathrm{N}^{-1}$. C ( $(\mathrm{t})$ and $\mathrm{c}(\mathrm{t})$, and $\zeta_{\mathrm{u}}(\mathrm{t})$ and $\xi_{\mathrm{u}}(\mathrm{t})$ for various values of $\mathrm{N}$ and $\mathrm{M}$ were difficult to distinguish in the figures. In this mesoscopic MD simulation using multiparticle collision dynamics, the system is divided into several cells in which the mass, momentum and energy are conserved. While the solvent particles only near the BP interact with it, the other particles far from the BP are momentum-conserved in each cell. Since the momentum of the BP is defined as the negative of the total momentum of the solvent particles, the decay of $\mathrm{C}(\mathrm{t})$ is very slow for large $\mathrm{N}$. In the present MD simulation study, the solvent particles interact with each other and the momentum of the solvent particles far from the BP is in question. If we neglect the interaction between solvent particles, then only short CWA interactions between the BP and the near solvent particles exist, and the other particles far from the BP undergo free streaming motion. In this case, we expect that the decay of $C(t)$ is very slow for large $N$. This test system is currently under study.

We then conclude the discussion of our results in Fig. 3, which show the logarithm of the time dependent friction coefficient $\zeta_{\mathrm{u}}(\mathrm{t})$ and momentum autocorrelation function $\mathrm{C}(\mathrm{t})$ for the case of $S 2 / 2$. The dotted line is the logarithm of $\zeta_{u}(t)$ calculated directly from Eq. (6) (the dashed line in Fig. 2), and the straight line is the extrapolation of the exponential long timedecay to $\mathrm{t}=0$ and is used to determine $\zeta_{1}$ and $\zeta_{2}$, according to Eq. (5). The dashed line (the dashed line in Fig. 1) is that of $\mathrm{C}(\mathrm{t})$ calculated directly from Eq. (7) and is used to determine $\zeta_{3}$. The dot-dashed line is $\xi_{\mathrm{u}}(\mathrm{t})$ obtained by time differentiation of the dashed line $\mathrm{C}(\mathrm{t})$ using Eq. (8), and the straight line is the extrapolation of the exponential long time decay to $t=0$ and is 
Table 2. Comparison of friction coefficients $\zeta_{\mathrm{i}}(\mathrm{kg} / \mathrm{ps} \cdot \mathrm{mol})$ of the Brownian particle. $\zeta_{1}^{*}$ is the reduced friction coefficient of $\zeta_{1}$, defined by $\zeta_{1}^{*}=\zeta_{1} \sigma_{\mathrm{B}}(\mathrm{m} \epsilon)^{-1 / 2}$

\begin{tabular}{llccccc}
\hline System & \multicolumn{1}{c}{$\zeta_{1}\left(\zeta_{1}^{*}\right)$} & $\zeta_{2}$ & $\zeta_{3}$ & $\zeta_{4}$ & $\zeta_{5}$ & $\zeta_{6}$ \\
\hline S2/1 & $0.732(73.2)$ & 0.161 & 0.801 & 1.091 & 2.965 & 0.756 \\
S2/2 & $0.167(16.7)$ & 0.474 & 0.588 & 1.334 & 3.353 & 0.158 \\
S2/4 & $0.042(4.2)$ & 0.320 & 0.512 & 0.750 & 3.089 & 0.044 \\
S4/2 & $0.369(73.8)$ & 1.086 & 0.759 & 1.357 & 2.542 & 0.363 \\
S4/4 & $0.094(18.8)$ & 0.376 & 0.674 & 0.932 & 3.081 & 0.091 \\
S4/8 & $0.021(4.2)$ & 0.041 & 0.637 & 0.970 & 3.514 & 0.022 \\
S8/4 & $0.180(72.0)$ & 0.659 & 0.648 & 1.190 & 4.140 & 0.174 \\
S8/8 & $0.043(17.2)$ & 0.163 & 0.371 & 0.476 & 1.455 & 0.044 \\
S8/16 & $0.011(4.4)$ & 0.321 & 0.295 & 0.548 & 3.619 & 0.010 \\
\hline
\end{tabular}

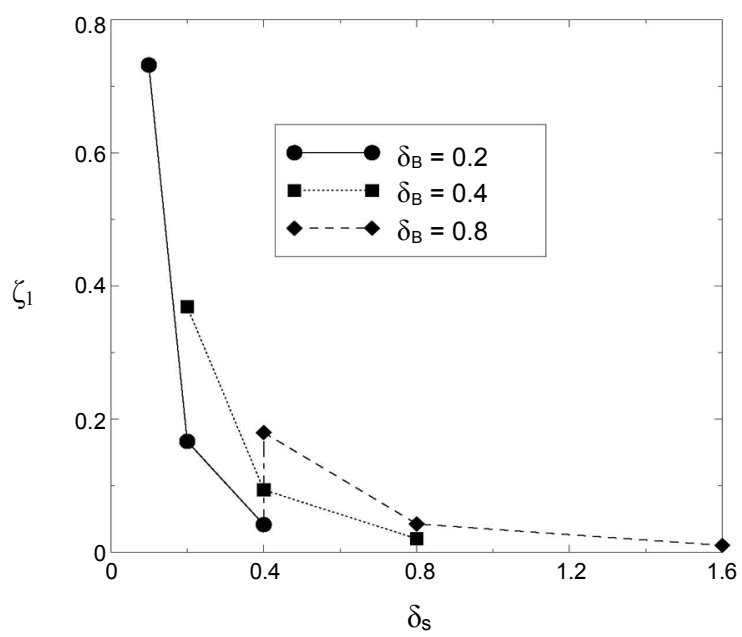

Figure 4. Friction coefficient $\zeta_{1}$ as function of $\sigma_{\mathrm{s}}$ for a given $\sigma_{\mathrm{B}}$. The dot-dashed line indicates the change of $\zeta_{1}$ with the increase of $\sigma_{\mathrm{B}}$ for $\sigma_{\mathrm{s}}=0.4 \mathrm{~nm}$.

used to determine $\zeta_{4}$ and $\zeta_{5}$. The long-dashed line (the longdashed line in Fig. 1) is $\mathrm{c}(\mathrm{t})$ obtained by the time integration of the dotted line $\zeta_{\mathrm{u}}(\mathrm{t})$ using Eq. (9) to determine $\zeta_{6}$. It is notable that the behavior of the dot-dashed line $\xi_{\mathrm{u}}(\mathrm{t})$ has a similarity to that of the dotted line $\zeta_{\mathrm{u}}(\mathrm{t})$ with a multiplying factor. If the momentum autocorrelation function $\mathrm{C}(\mathrm{t})$ calculated directly from Eq. (7) is correct, then $\xi_{\mathrm{u}}(\mathrm{t})$ obtained by time differentiation of $\mathrm{C}(\mathrm{t})$ using Eq. (8) should be the same as $\zeta_{\mathrm{u}}(\mathrm{t})$ calculated directly from Eq. (6). That is, $\mathrm{C}(\mathrm{t})$ is not small enough to encounter the factor $\mathrm{Nm}(=127.84 \mathrm{~kg} / \mathrm{mol})$ in Eq. (8) for some reason. Likewise, since $\zeta_{\mathrm{u}}(\mathrm{t})$ calculated directly from Eq. (6) is correct, $\mathrm{c}(\mathrm{t})$ obtained by the time integration of $\zeta_{\mathrm{u}}(\mathrm{t})$ using Eq. (9) is correct. In order for $\mathrm{C}(\mathrm{t})$ to be the same to $\mathrm{c}(\mathrm{t}), \mathrm{C}(\mathrm{t})$ should be reduced in proportion to the ratio of the slopes $\zeta_{6} / \zeta_{3}=$ $0.158 / 0.588=0.27$, to encounter the factor $\mathrm{Nm}$ in Eq. (8).

The estimated friction coefficients for all the systems obtained from our momentum-conserved MD simulations are collected in Table 2. We have found that it is difficult to determine the friction coefficient from the slope, $-\zeta_{2} / \mathrm{Nm}$, of $\zeta_{\mathrm{u}}(\mathrm{t})$, especially for very large $\mathrm{N}$ where the slope is close to zero. While the slopes scale as $1 / \mathrm{N}$ for the smaller $\mathrm{N}$ values, the small value of the slope and relatively large statistical error makes it difficult to determine this scaling for very large values of $\mathrm{N}$. However, $\zeta_{1}$ may be determined accurately from the extrapola- tion of the long time behavior of $\zeta_{\mathrm{u}}(\mathrm{t})$ to $\mathrm{t}=0$ as discussed above. The estimated friction coefficient from the slope, $-\zeta_{6} / \mathrm{Nm}$, of $\mathrm{c}(\mathrm{t})$ also gives a reasonable value since the $\mathrm{c}(\mathrm{t})$ is obtained by time integration of $\zeta_{\mathrm{u}}(\mathrm{t})$ using Eq.(9). The other friction coefficients $\zeta_{3}, \zeta_{4}$, and $\zeta_{5}$, which are obtained from $\mathrm{C}(\mathrm{t})$ directly using Eq. (7) and obtained from $\xi_{\mathrm{u}}(\mathrm{t})$ by time integration of $\mathrm{C}(\mathrm{t})$ using Eq. (8), are not correct since the momentum autocorrelation function $\mathrm{C}(\mathrm{t})$ is not correct as discussed above.

Fig. 4 shows the friction coefficient $\zeta_{1}$ as a function of $\sigma_{\mathrm{s}}$ for different $\sigma_{\mathrm{B}}$. For a given $\sigma_{\mathrm{B}}$, the friction coefficient decreases quadratically with $\sigma_{\mathrm{s}}$. In other words, the friction coefficient increases quadratically with the ratio of $\sigma_{\mathrm{B}} / \sigma_{\mathrm{s}}$ for all $\sigma_{\mathrm{B}}$. A rough estimate of the microscopic contribution to the friction can be obtained using a hard sphere binary collision model with the collision diameter chosen to be $\sigma_{\mathrm{B}}: \zeta_{\mathrm{m}}=8 / 3 \rho \sigma_{\mathrm{B}}^{2}(2 \pi \mathrm{mkT})^{1 / 2}$. The behavior of $\zeta_{1}$ on the ratio of $\sigma_{\mathrm{B}} / \sigma_{\mathrm{s}}$ follows the microscopic description of the friction. On the other hand, for a given $\sigma_{\mathrm{s}}$, for example for $\sigma_{\mathrm{s}}=0.4 \mathrm{~nm}$, the friction coefficient increases linearly with $\sigma_{B}$ along the dot-dashed line in Fig. 4 . The hydrodynamic contribution of the friction is given by $\zeta_{\mathrm{h}}=4 \pi \sigma_{\mathrm{B}} \eta$ with the slip boundary condition. In this case, the behavior of $\zeta_{1}$ on $\sigma_{\mathrm{B}}$ for a given $\sigma_{\mathrm{s}}$ follows the hydrodynamic description of the friction. Usually the microscopic and hydrodynamic descriptions of the friction are adequate for small and large $\sigma_{\mathrm{B}}$, respectively. ${ }^{5}$ Finally the reduced friction coefficients, $\zeta_{1}^{*}$, are listed in Table 2 and $\zeta_{1}^{*}$ for the same ratio of $\sigma_{\mathrm{B}} / \sigma_{\mathrm{S}}$ are essentially the same, which confirms the validity of the estimate of $\zeta_{1}$ through our MD simulations.

\section{Conclusion}

The long time molecular dynamics (MD) simulations of both the momentum autocorrelation functions and the time dependent friction coefficients of a Brownian particle (BP) in a microcanonical ensemble are carried out to determine the friction coefficient of the BP. Although many MD simulation methods are employed to treat the infinite mass limit of the Brownian particle, the determination of the friction coefficients from the decay rates of the momentum autocorrelation functions is difficult due to the invalid momentum conservation of the system in both the infinite mass and the constraint MD simulations and due to the fast decay rates of these correlation functions in the momentum-conserved MD simulation. Also, the determination of the friction coefficients from the slopes of the time dependent friction coefficients, especially for very large $\mathrm{N}$ where the slope is close to zero, is difficult due to the scaling of the slope as $1 / \mathrm{N}$. However, the friction coefficient $\left(\zeta_{1}\right)$ can be determined correctly from the time dependent friction coefficient by measuring the extrapolation of its long time decay to $t=0$. The momentum autocorrelation function obtained by time integration of the time dependent friction coefficient, which is calculated from the momentum-conserved MD simulation, can also give a correct estimate $\left(\zeta_{6}\right)$ of the friction coefficient from its decay rate. The friction coefficients $\zeta_{6}$ and $\zeta_{1}$ are in a good accord since the calculation of the time dependent friction coefficients for the momentum-conserved MD simulations is valid, and the momentum autocorrelation function is obtained by time integration of time dependent friction coefficient. The 
other friction coefficients $\zeta_{2}, \zeta_{3}, \zeta_{4}$, and $\zeta_{5}$ are not correct as discussed in the previous section.

While the friction coefficient increases quadratically with the ratio of $\sigma_{\mathrm{B}} / \sigma_{\mathrm{s}}$ for all $\sigma_{\mathrm{B}}$, which follows the microscopic description of the friction: $\zeta_{\mathrm{m}}=8 / 3 \rho \sigma_{\mathrm{B}}^{2}(2 \pi \mathrm{mkT})^{1 / 2}$, for a given $\sigma_{\mathrm{s}}$ the friction coefficient increases linearly with $\sigma_{\mathrm{B}}$ which follows the hydrodynamic description of the friction, $\zeta_{\mathrm{h}}=4 \pi \sigma_{\mathrm{B}} \eta$. Finally it is found that the reduced friction coefficients $\zeta_{1}^{*}$ for the same ratio of $\sigma_{\mathrm{B}} / \sigma_{\mathrm{s}}$ are essentially the same.

Acknowledgments. This research was supported by Kyungsung University Research Grants in 2010.

\section{References}

1. Kubo, R. J. Phys. Soc. Jpn. 1957, 12, 570; Kubo, R. Rep. Prog.
Phys. 1966, 29, 255; Kubo, R. In Many-Body Problems, The Fluctuation Dissipation Theorem; Parry, W. E. et al. Eds.; Benjamin: New York, 1969; 235.

2. Brey, J. J.; Gómez Ordóñez, J. J. Chem. Phys. 1982, 76, 3260.

3. Español, P.; Zúñiga, I. J. Chem. Phys. 1993, 98, 574.

4. Ould-Kaddour, F.; Levesque, D. J. Chem. Phys. 2003, 118, 7888.

5. Lee, S. H.; Kapral, R. J. Chem. Phys. 2004, 121, 11163.

6. Weeks, J. D.; Chandler, D.; Andersen, H. C. J. Chem. Phys. 1971, $54,5237$.

7. Swope, W. C.; Andersen, H. C.; Berens, P. H.; Wilson, K. R. J. Chem. Phys. 1982, 76, 637.

8. Hansen, P.; McDonald, I. R. Theory of Simple Liquids; Academic: New York, 1986.

9. Lebowitz, J. L.; Rubin, E. Phys. Rev. 1963, 131, 2381.

10. Résibois, P.; Davis, H. T. Physica 1964, 30, 1077.

11. Lebowitz, J. L.; Résibois, P. Phys. Rev. 1965, 139, 1101.

12. Mazur, P.; Oppenheim, I. Physica 1970, 50, 241.

13. Ryckaert, J. P.; Ciccotti, G.; Berendsen, H. J. J. Comp. Phys. 1977, 23,327 . 